\title{
SuR UnE GÉNÉRALISATION DE LA NOTION DE $V$-VARIÉTÉ
}

\section{Elisa PRATO}

Laboratoire Dieudonné, Université de Nice, Parc Valrose, 06108 Nice Cedex 2, France

Courriel: elisa@math.unice.fr

Web: http://www-math.unice.fr/ ${ }^{\sim}$ elisa/home.html

Résumé. Nous considérons un espace topologique qui est localement isomorphe au quotient de $\mathbb{R}^{k}$ par l'action d'un groupe discret et nous l'appelons quasi-variété de dimension $k$. Les quasivariétés généralisent les variétés et les $V$-variétés et représentent le cadre naturel pour la réduction symplectique par rapport à l'action induite d'un sous-groupe de Lie, compact ou non, d'un tore. Nous définissons les quasi-tores, les actions hamiltoniennes de quasi-tores et l'application moment sur une quasi-variété symplectique, et nous montrons que tout polytope convexe simple, rationnel ou non, est l'image de l'application moment pour l'action d'un quasi-tore sur une quasi-variété.

\section{ON A GENERALIZATION OF THE NOTION OF ORBIFOLD}

Abstract. We consider a topological space which is locally isomorphic to the quotient of $\mathbb{R}^{k}$ by the action of a discrete group and we call it quasifold of dimension $k$. Quasifolds generalize manifolds and orbifolds and represent the natural framework for performing symplectic reduction with respect to the induced action of any Lie subgroup, compact or not, of a torus. We define quasitori, Hamiltonian actions of quasitori and the moment mapping for symplectic quasifolds, and we show that every simple convex polytope, rational or not, is the image of the moment mapping for the action of a quasitorus on a quasifold.

\section{Introduction}

Soit $M$ une variété symplectique compacte et connexe et soit $T$ un tore agissant sur $M$ de façon hamiltonienne. Alors, d'après le théorème de convexité d'Atiyah et Guillemin-Sternberg [1], 6], l'image de l'application moment correspondante est un polytope convexe rationnel. Si l'action de $T$ est effective et $\operatorname{dim} M=2 \operatorname{dim} T$ alors, d'àpres un théorème de Delzant [4], le polytope image est un polytope rationnel simple satisfaisant à une certaine condition d'intégralité, et ce polytope détermine la variété à symplectomorphisme équivariant près. Au cours de la démonstration de ce théorème, Delzant donne une construction explicite de la variété à partir du polytope; cette construction se base sur la technique de réduction symplectique. Les méthodes d'Atiyah, GuilleminSternberg et Delzant ont succesivement étés adaptées par Lerman-Tolman [9] au cas des $V$-variétés, mais les polytopes image, obtenus dans ce cas, sont toujours des polytopes rationnels. Il est d'ailleurs très naturel de se demander si tout polytope simple, rationnel ou non, est l'image de l'application moment pour un espace symplectique adéquat. Pour pouvoir répondre à cette question nous considérons des espaces topologiques qui généralisent les variétés et les $V$-variétés, mais qui ne sont plus forcement des espaces séparés. Ces espaces, que nous appelons quasi-variétés, permettent l'utilisation de la technique de réduction symplectique sous des hypothèses assez faibles; par conséquent, ils donnent le moyen d'étendre la construction de Delzant au cas des polytopes non rationnels. L'unicité dans le théorème de Delzant n'est plus valable dans ce contexte (voir exemple 9). Dans le cas où $\Delta$ est rationnel cette construction nous donne une famille de $V$-variétés, en accord avec [9]; si $\Delta$ vérifie aussi la condition d'intégralité de Delzant, on trouve parmi cette famille une vraie variété, en accord avec $\llbracket$. 
Remarquons que l'espace des orbites de l'action d'un groupe discret sur une variété a été etudié par Connes [3, chapitre II] dans le cadre de la géométrie non commutative. Les quasi-variétés sont aussi reliées à la géométrie des quasi-cristaux [1].

Nous renvoyons le lecteur à 10 pour les preuves des résultats énoncés, ainsi que pour de nombreux exemples; dans une suite à [10] on trouvera un traitement plus detaillé des propriétés de convexité de l'application moment. Finalement dans un travail en collaboration avec Battaglia [2] nous introduisons des structures complexes et des structures de Kähler sur les quasi-variétés, et nous montrons que les espaces du théorème 11 peuvent être considérés comme la généralisation naturelle des variétés toriques qu'on associe à tout polytope convexe simple qui est rationnel.

\section{Définitions et résultats}

Commençons par donner la définition de modèle de quasi-variété et de difféomorphisme entre modèles.

Définition 1 (Modèle) Soit $\tilde{U}$ une variété connexe de dimension $k$ et soit $\Gamma$ un groupe discret agissant différentiablement sur $\tilde{U}$ de façon que l'ensemble des points où l'action est libre, soit connexe et dense. Un modèle de dimension $k, \tilde{U} / \Gamma$, est l'espace des orbites de l'action de $\Gamma$ sur $\tilde{U}$, muni de la topologie quotient.

On peut toujours supposer, en passant éventuellement au revêtement universel, que $\tilde{U}$ soit simplement connexe, ce que nous ferons par la suite.

Définition 2 (Difféomorphisme de modèles) Soient $p: \tilde{U} \rightarrow \tilde{U} / \Gamma$ et $q: \tilde{V} \rightarrow \tilde{V} / \Delta$ deux modèles. On dira qu'une application $f: \tilde{U} / \Gamma \longrightarrow \tilde{V} / \Delta$ est un difféomorphisme de modèles s'il existe un difféomorphisme $\tilde{f}: \tilde{U} \longrightarrow \tilde{V}$ et un isomorphisme $F: \Gamma \rightarrow \Delta$ tels que $q \circ \tilde{f}=f \circ p$ et $\tilde{f}(\gamma \cdot-)=$ $F(\gamma) \cdot \tilde{f}(-), \gamma \in \Gamma$. Nous dirons alors que $\tilde{f}$ est un relevé de $f$.

On montre aisement que deux relevés d'un difféomorphisme $f$ coïncident à moins d'une multiplication à droite par un élément de $\Gamma$ (ou à gauche par un élément de $\Delta$ ). Nous pouvons maintenant définir une quasi-variété à partir des deux définitions précédentes.

Définition 3 (Quasi-variété) Soit $M$ un espace topologique. Un atlas de quasi-variété de dimension $k$ sur $M$ est une collection d'ouverts $\mathcal{A}=\left\{U_{\alpha} \mid \alpha \in A\right\}$, dits cartes, ayant les propriétés suivantes:

1. les $U_{\alpha}$ recouvrent $M$;

2. pour tout $\alpha \in A$ il existe un modèle $\tilde{U}_{\alpha} / \Gamma_{\alpha}$, où $\tilde{U}_{\alpha}$ est un sous-ensemble ouvert, connexe et simplement connexe de $\mathbb{R}^{k}$, et un homéomorphisme $\phi_{\alpha}: \tilde{U}_{\alpha} / \Gamma_{\alpha} \longrightarrow U_{\alpha}$;

3. pour tous $\alpha, \beta \in A$ tels que $U_{\alpha} \cap U_{\beta} \neq \emptyset$ l'application

$$
g_{\alpha \beta}=\phi_{\beta}^{-1} \circ \phi_{\alpha}: \phi_{\alpha}^{-1}\left(U_{\alpha} \cap U_{\beta}\right) \longrightarrow \phi_{\beta}^{-1}\left(U_{\alpha} \cap U_{\beta}\right)
$$

est un difféomorphisme de modèles. Nous dirons alors que $g_{\alpha \beta}$ est un changement de cartes.

Deux atlas sur $M$ sont équivalents si l'union des leurs cartes est encore un atlas de $M$. Une structure de quasi-variété sur $M$ est une classe d'équivalence d'atlas; nous dirons alors qu'un espace $M$ avec une structure de quasi-variété est une quasi-variété.

Si les groupes $\Gamma_{\alpha}$ sont finis on retrouve ici la définition classique de $V$-variété, s'ils sont triviaux on retrouve la définition de vraie variété. 
Exemple 4 (Quasi-sphère) Soient $s, t$ deux nombres réels positifs tels que $s / t \notin \mathbb{Q}$. Considérons $\mathbb{C}^{2}$ avec sa forme symplectique canonique $\omega_{0}=\frac{1}{2 \pi i}\left(d z_{1} \wedge d \bar{z}_{1}+d z_{2} \wedge d \bar{z}_{2}\right)$ et l'action de $\mathbb{R}:\left(\theta,\left(z_{1}, z_{2}\right)\right)=\left(e^{2 \pi i \theta} z_{1}, e^{2 \pi i \theta \frac{s}{t}} z_{2}\right)$ d'application moment $\Psi\left(z_{1}, z_{2}\right)=\left|z_{1}\right|^{2}+\frac{s}{t}\left|z_{2}\right|^{2}-s$. Considérons le niveau régulier $\Psi^{-1}(0)$; alors l'espace des orbites $M=\Psi^{-1}(0) / \mathbb{R}$ est une quasi-variété de dimension 2 .

Soit $M$ une quasi-variété. Pour définir une géométrie sur $M$ nous procédons de la façon suivante: d'une manière générale, un objet géométrique sur une quasi-variété est la donnée d'un objet géométrique sur tout ouvert $\tilde{U}_{\alpha}$, invariant par rapport à l'action du groupe discret $\Gamma_{\alpha}$ et satisfaisant à des conditions de compatibilité. Par exemple, une forme différentielle de degré $h, \omega$, sur $M$ est la donnée, pour tout $\alpha \in \mathcal{A}$, d'une forme différentielle $\Gamma_{\alpha}$-invariante de degré $h, \tilde{\omega}_{\alpha}$, sur $\tilde{U}_{\alpha}$ qui se transforme de manière compatible aux changements de cartes $g_{\alpha \beta}$; une 2-forme est dite symplectique si toute $\tilde{\omega}_{\alpha}$ est symplectique (fermée et non-dégénérée). Nous renvoyons le lecteur à l'article [10] pour les définitions de: champ de vecteurs, application différentiable, difféomorphisme, image réciproque d'une forme, image directe d'un champ de vecteurs, différentiel et produit intérieur. L'analogue du tore dans la géométrie des quasi-variétés s'appelle quasi-tore. Soit $\mathfrak{d}$ un espace vectoriel de dimension $n$.

Définition 5 (Quasi-réseau, quasi-tore, quasi-algèbre de Lie) Un quasi-réseau, $Q$, dans $\mathfrak{d}$ est le $\mathbb{Z}$-module engendré par un ensemble de vecteurs $X_{1}, \ldots, X_{d}$ qui engendrent $\mathfrak{d}$. Nous appelons quasi-tore de dimension $n$ le groupe quotient $D=\mathfrak{d} / Q$. La quasi-algèbre de Lie du quasi-tore $D$ est l'espace vectoriel $\mathfrak{d}$.

Un quasi-tore est evidemment une quasi-variété et les operations de groupe sont différentiables. Si $Q$ est un vrai réseau (par exemple si $d=n$ ) on retrouve dans la définition précédente le tore et son algèbre de Lie. Les quasi-tores de dimension un ont étés étudiés par Donato, Iglesias et Lachaud [5, 7, 8]; Iglesias introduit à cette occasion la terminologie tores irrationnels.

Exemple 6 (Quasi-cercle) Considérons dans $\mathbb{R}$ le quasi-réseau $Q=s \mathbb{Z}+t \mathbb{Z}, s / t \notin \mathbb{Q}$. Alors $D^{1}=\mathfrak{d} / Q$ est un quasi-tore de dimension 1 .

Les quasi-tores apparaissent de façon très naturelle, comme le montre la proposition suivante.

Proposition 1 Soit $T$ un tore et soit $N$ un sous-groupe de Lie de $T$. Alors $T / N$ est un quasi-tore de dimension $n=\operatorname{dim} T-\operatorname{dim} N$.

Par exemple le quotient du tore de dimension 2 par une droite de pente irrationnelle $s / t \notin \mathbb{Q}$ est le quasi-tore de l'exemple 6 .

Définition 7 (Action différentiable) Une action différentiable d'un quasi-tore $D$ sur une quasivariété $M$ est une application différentiable $\tau: D \times M \longrightarrow M$ telle que $\tau\left(d_{1} \cdot d_{2}, m\right)=\tau\left(d_{1}, \tau\left(d_{2}, m\right)\right)$, et $\tau\left(1_{D}, m\right)=m$ pout tout $d_{1}, d_{2} \in D$ et $m \in M$.

Etant donné une action différentiable d'un quasi-tore sur une quasi-variété il est toujours possible d'associer à tout $X \in \mathfrak{d}$, comme dans le cas des variétés, un champ de vecteurs sur $M$, dit champ fondamental de l'action, noté $\mathrm{X}_{M}$.

Définition 8 (Action hamiltonienne, application moment) Une action différentiable d'un quasi-tore $D$ sur une quasi-variété $M$ est dite hamiltonienne si elle respecte la forme symplectique et s'il existe une application différentiable $D$-invariante $\Phi: M \longrightarrow \mathfrak{d}^{*}$, dite application moment, ayant la propriété que $\imath\left(\mathrm{X}_{M}\right) \omega=d<\Phi, X>$, pour tout $X \in \mathfrak{d}$. 
Exemple 9 Considérons la quasi-sphère de l'exemple 4 et le quasi-cercle de l'exemple 6. L'application $\tau([\theta],[z: w])=\left[e^{\frac{2 \pi i \theta}{s}} z: w\right]$ définit une action hamiltonienne de $D^{1}$ sur $M$, d'application moment $\Phi([z: w])=\frac{|z|^{2}}{s}=1-\frac{|w|^{2}}{t}$. Remarquons que $\Phi(M)=[0,1]$ (indepéndamment de $s$ et $t$ ) comme pour la rotation de la sphère de $\mathbb{R}^{3}$ autour de l'axe $O z$.

Pour obtenir beaucoup d'autres exemples d'actions hamiltoniennes de quasi-tores sur des quasivariétés il suffit de prendre la réduction symplectique par rapport à un sous-groupe de Lie d'un vrai tore agissant sur une vraie variété symplectique.

Théorème 10 (Réduction) Soit $T$ un tore d'algèbre de Lie $\mathfrak{t}$, soit $T \times X \longrightarrow X$ une action hamiltonienne de $T$ sur une variété symplectique $X$ et supposons que l'application moment $J: X \longrightarrow \mathfrak{t}^{*}$ soit propre. Considérons l'action induite d'un sous-groupe de Lie $N$ de $T$ et supposons que 0 soit une valeur régulière de l'application moment correspondante: $\psi=i^{*} \circ J: X \longrightarrow \mathfrak{n}^{*}(\mathfrak{n}$ dénote l'algèbre de Lie de $N$ et $i$ l'inclusion dans $\mathbf{t}$.) Alors $M=\psi^{-1}(0) / N$ est une quasi-variété symplectique de dimension $\operatorname{dim} X-2 \operatorname{dim} N$ et l'action induite de $T / N$ sur $M$ est hamiltonienne.

Ce dernier théorème permet d'étendre la construction de Delzant et donc de montrer que tout polytope simple $\Delta \subset \mathfrak{d}^{*}$ est l'image par l'application moment d'une quasi-variété. Rappelons qu'un polytope convexe est dit simple si de chaque sommet sont issues exactement $n$ arêtes (ici nous supposons par simplicité $\operatorname{dim} \Delta=n)$. Nous avons alors:

Théorème 11 Pour tout polytope convexe simple $\Delta \subset \mathfrak{d}^{*}$ il existe un quasi-tore de dimension $n$ et de quasi-algèbre de Lie $\mathfrak{d}, D$, une quasi-variété symplectique compacte de dimension $2 n$, $M$, et une action hamiltonienne effective de $D$ sur $M$ telle que l'image de l'application moment correspondante soit $\Delta$.

\section{Références bibliographiques}

[1] M. Atiyah, Convexity and commuting Hamiltonians, Bull. London Math. Soc. 14 (1982), $1-15$.

[2] F. Battaglia and E. Prato, Simple non-rational convex polytopes and Kähler structures, en cours de rédaction.

[3] A. Connes, Noncommutative Geometry, Acad. Press (1994).

[4] T. Delzant, Hamiltoniens périodiques et image convexe de l'application moment, Bull. S.M.F. 116 (1988), 315-339.

[5] P. Donato et P. Iglesias, Exemples de groupes difféologiques: flots irrationnels sur le tore, $C$. R. Acad. Sci. Paris 301 (1985) 127-130.

[6] V. Guillemin and S. Sternberg, Convexity properties of the moment mapping, Invent. Math. 67 (1982), 491-513.

[7] P. Iglesias, Fibrations difféologiques et Homotopie, Thèse de Doctorat, Université de Provence (1985).

[8] P. Iglesias et G. Lachaud, Espaces différentiables singuliers et corps de nombres algébriques, Ann. Inst. Fourier, Grenoble 40, 1 (1990), 723-737.

[9] E. Lerman and S. Tolman, Hamiltonian torus actions on symplectic orbifolds and toric varieties, Trans. A.M.S. 349 No. 10 (1997), 4201-4230.

[10] E. Prato, Simple non-rational convex polytopes via symplectic geometry, prépublication (1998).

[11] M. Senechal, Quasicrystals and geometry, Cambridge U. Press (1985). 